\title{
Torsion and Burgers vector of a tube dislocation
}

\section{Mikhail Katanaev*}

Steklov Mathematical Institute, ul. Gubkina, 8, Moscow, 119991, Russia

E-mail: katanaev@mi.ras.ru

We consider recently discovered new exact solution of three dimensional Euclidean Einstein's equations corresponding to massive thin cylindrical shell (tube) with $\delta^{\prime}$-type source. The metric components are discontinuous functions but all ambiguous terms in Einstein's equations cancel. The solution describes a tube dislocation. The components of torsion tensor and Burgers vector characterizing this defect are computed.

Corfu Summer Institute on Elementary Particles and Physics - Workshop on Non Commutative Field Theory and Gravity,

September 8-12, 2010

Corfu Greece

\footnotetext{
*Speaker.
} 


\section{Introduction}

Recently, a new exact solution of three dimensional Euclidean Einstein's equations was found [1]. The metric components are discontinuous functions but surprisingly all ambiguous terms in Einstein's equations cancel. This solution corresponds to $\delta^{\prime}$-type energy-momentum tensor. The metric has straightforward physical interpretation as describing a tube dislocation, and can be obtained within the ordinary elasticity theory.

The presence of a tube dislocation in media results in changing of three dimensional space metric. It means that the Schrödinger equation describing the motion of a quantum particle in a space with defect should be modified not only by some potential term but also by the kinetic term. This idea was used to describe motion of a quantum particle in a double wall nanotube [2]. We showed that modification of a kinetic term in the Schrödinger equation by the metric for a tube dislocation changes the spectrum of a quantum particle.

Originally, we computed curvature tensor components for a tube dislocation and showed that all ambiguous terms (like the product of a step and $\delta$ functions) disappear in Einstein's equations. The net result is the $\delta^{\prime}$-function in the right hand side. At the same time, existence of a dislocation leads to the appearance of nontrivial torsion in space [3-7] (for review, see [8]). This fact is well known in General Relativity which can be equivalently reformulated in a space-time with absolute parallelism (zero curvature) but nontrivial torsion. Here we perform this reformulation for a tube dislocation and compute the Burgers vector which is the main characteristic of a dislocation.

\section{Tube dislocation in the linear elasticity theory}

We consider three-dimensional Euclidean space $\mathbb{R}^{3}$ (infinite homogeneous and isotropic elastic media or eather in general relativity) with Cartesian coordinates $x^{i}, y^{i}, i=1,2,3$, and Euclidean metric $\delta_{i j}=\operatorname{diag}(+++)$. The basic variable in the elasticity theory is the displacement vector field $u^{i}(x), x \in \mathbb{R}^{3}$, which measures the displacement of a point in the elastic media. In the absence of external forces, Newton's and Hooke's laws in the linear approximation reduce to three second order partial differential equations which describe the equilibrium state of elastic media (see, i.e. Ref. [9]),

$$
(1-2 \sigma) \triangle u_{i}+\partial_{i} \partial_{j} u^{j}=0
$$

where $\sigma$ is the dimensionless Poisson ratio characterizing the elastic properties of media and $\triangle$ is the Laplace operator. Raising and lowering of Latin indices $i, j, \ldots$ is performed using the Euclidean metric $\delta_{i j}$ and its inverse $\delta^{i j}$. The boundary conditions for Eq.(2.1) correspond to the physical problem which is to be solved.

Let us pose the problem for the tube dislocation shown in Fig.1 $(a)$. This dislocation is produced as follows. We cut out the thick cylinder of media located between two parallel cylinders of radii $r_{1}$ and $r_{2}\left(r_{1}<r_{2}\right)$ with axis $z=x^{3}$ as the axis of both cylinders, move symmetrically both cutting surfaces one to the other, and afterwards glue them. In the equilibrium state, the gluing surface is also the cylinder due to circular and translational symmetries of the problem of radius $r_{*}$ which is to be found. We denote the thickness of the removed tube by $l=r_{2}-r_{1}$. 

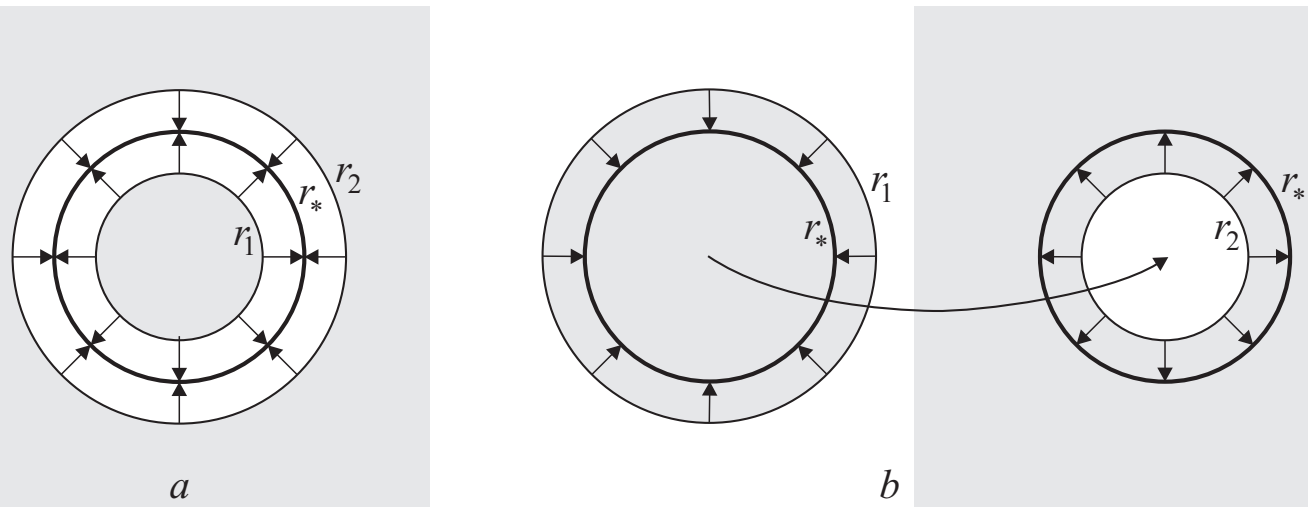

Figure 1: Negative $(a)$ and positive $(b)$ tube dislocations.

In our conventions, Fig.1 $(a)$ shows the negative tube dislocation because part of the media was removed, $r_{1}<r_{2}$. This procedure can be inverted by addition of extra media to $\mathbb{R}^{3}$ as shown in Fig.1(b). In this case, we call it positive tube dislocation, $r_{1}>r_{2}$.

This problem is naturally formulated and solved in cylindrical coordinates $r, \varphi, z$. Let us denote the displacement field components in cylindrical coordinates by $u^{r}, u^{\varphi}, u^{z}$. In our case, $u^{\varphi}=0$, $u^{z}=0$, due to the symmetry of the problem, and the radial displacement field $u^{r}(r)$ depends only on the radius $r$, and we drop the index, $u^{r}(r)=u(r)$, for simplicity.

The boundary conditions for the tube dislocation are

$$
\left.u_{\text {in }}\right|_{r=0}=0,\left.\quad u_{\mathrm{ex}}\right|_{r=\infty}=0,\left.\quad u_{\mathrm{in}}\right|_{r=r_{*}}-\left.u_{\mathrm{ex}}\right|_{r_{*}}=l,\left.\quad \frac{d u_{\mathrm{in}}}{d r}\right|_{r=r_{*}}=\left.\frac{d u_{\mathrm{ex}}}{d r}\right|_{r=r_{*}} .
$$

The first three conditions are purely geometrical, and the third one means the equality of normal elastic forces inside and outside the gluing surface in the equilibrium state. The subscripts "in" and "ex" denote the displacement vector field inside and outside the gluing surface, respectively.

Our definition of the displacement vector field differs slightly from the usual one. In our notations, the point with coordinates $y^{i}$ after elastic deformation moves to the point with coordinates $x^{i}$

$$
y^{i} \mapsto x^{i}(y)=y^{i}+u^{i}(x),
$$

the displacement vector field being the difference between new and old coordinates, $u^{i}(x)=x^{i}-y^{i}$ (this is usual). The difference is that we consider the components of the displacement vector field $u^{i}(x)$ as functions of the final state coordinates of media points $x^{i}$ and not of the initial ones $y^{i}$. This is equivalent to the standard approach in the absence of dislocations because both sets of coordinates $x^{i}$ and $y^{i}$ cover the entire Euclidean space $\mathbb{R}^{3}$. If the dislocation is present, the final state coordinates $x^{i}$ cover the whole $\mathbb{R}^{3}$ while the initial state coordinates cover only part of the Euclidean space lying outside the thick cylinder which was removed. Therefore the final state coordinates are preferable in the presence of dislocations. This difference can be considered as inessential in the linear approximation but the geometric theory of defects describes large deformations along with the small ones. 
The elasticity equations (2.1) with boundary conditions (2.2) were solved in [1]. The resulting displacement vector field has only radial component

$$
u(r)=\left\{\begin{aligned}
\frac{l r}{2 r_{*}}, & r<r_{*}, \\
-\frac{l r_{*}}{2 r}, & r>r_{*} .
\end{aligned}\right.
$$

We note that the gluing surface is located exactly in the middle of two cylinders, $r_{*}=\left(r_{2}-\right.$ $\left.r_{1}\right) / 2$, and the solution does not depend on the Poisson ratio. This means that this defect has purely geometric origin.

The displacement vector field (2.4) describes diffeomorphism inside and outside the gluing surface and induces nontrivial metric

$$
g_{i j}(x)=\frac{\partial y^{k}}{\partial x^{i}} \frac{\partial y^{l}}{\partial x^{j}} \delta_{k l} .
$$

To calculate the metric in the whole space $\mathbb{R}^{3}$ we drop the $\delta$-function corresponding to the jump of the displacement vector field (2.4) on the gluing surface. This is due to the definition of the triad field in the geometric theory of defects [8]. The resulting metric has discontinuous angular component

$$
d s^{2}=(1-v)^{2} d r^{2}+(r-u)^{2} d \varphi^{2}+d z^{2}
$$

where

$$
v= \begin{cases}\frac{l}{2 r_{*}}, & r \leq r_{*}, \\ \frac{l r_{*}}{2 r^{2}}, & r \geq r_{*} .\end{cases}
$$

Metric (2.5) differs from the formal substitution of $y=r-u(r)$ in the Euclidean metric $d s^{2}=$ $d y^{2}+y^{2} d \varphi^{2}+d z^{2}$ by the square of the $\delta$-function in the $g_{r r}$ component. This procedure is a must in the geometric theory of defects, because otherwise the Burgers vector can not be expressed as the surface integral [8]. So, the metric component $g_{r r}(r)=(1-v)^{2}$ of the tube dislocation is a continuous function, and the angular component $g_{\varphi \varphi}=(r-u)^{2}$ has the jump across the cut.

The discontinuity of the angular metric component in (2.5) is unavoidable because the circumference of the circle has a jump across the cut, and it is a geometric invariant.

The components of the metric are not differentiable functions, and hence the calculation of geometric quantities involving derivatives and multiplications is an ambiguous procedure. Therefore, we perform all calculations as if the components were sufficiently smooth functions and see that all ambiguous terms safely cancel in the final answer. It means that whatever regularization of the components is applied the final answer does not depend on it.

In [1], we calculate Christoffel's symbols and curvature tensor components. They are not defined. At the same time, Einstein's equations,

$$
\sqrt{|g|}\left(\widetilde{R}_{\mu v}-\frac{1}{2} g_{\mu v} \widetilde{R}\right)=-\frac{1}{2} T_{\mu v}
$$

are identically satisfied with the zero right hand side except the $z z$ component,

$$
T_{z z}=\frac{4 l r_{*}}{2 r_{*}-l} \delta^{\prime}\left(r-r_{*}\right) \text {. }
$$


We see that all ambiguous terms cancel! The factor $\sqrt{|g|}$ appears in Einstein's equations (2.7) because $\delta$-function is not a function but a scalar density with respect to coordinate transformations.

\section{Torsion and Burgers vector}

The metric for a tube dislocation (2.5) does not describe the geometry of the elastic media, because any dislocation corresponds to nontrivial torsion [8]. Here we calculate torsion and the Burgers vector for a tube dislocation. Translational symmetry along $z$ axis reduces the problem to the $x, y$ plane. Let us mark the old Cartesian coordinates in the undeformed media before the dislocation is made by hat symbols $\left\{y^{a}\right\}=\hat{x}, \hat{y}, a=1,2$. New Cartesian and polar coordinates in the media with a tube dislocation are denoted by $\left\{x^{\alpha}\right\}=x, y$ and $r, \varphi, \alpha=1,2$. In the inside and outside regions old and new coordinates are related by the displacement vector field (2.4)

$$
\begin{array}{ll}
\hat{x}_{\mathrm{in}}=\left(1-\frac{l}{2 r_{*}}\right) r \cos \varphi, & \hat{x}_{\mathrm{ex}}=\left(r+\frac{l r_{*}}{2 r}\right) r \cos \varphi, \\
\hat{y}_{\mathrm{in}}=\left(1-\frac{l}{2 r_{*}}\right) r \sin \varphi, & \hat{y}_{\mathrm{ex}}=\left(r+\frac{l r_{*}}{2 r}\right) r \sin \varphi .
\end{array}
$$

According to a general prescription, the induced frame field in these regions is given by partial derivatives $e_{\alpha}^{a}=\partial_{\alpha} y^{a}$. Thus, we obtain

$$
\begin{array}{ll}
e_{(\mathrm{in}) r} r^{\hat{x}}=\left(1-\frac{l}{2 r_{*}}\right) \cos \varphi, & e_{(\mathrm{ex}) r^{\hat{x}}}=\left(1-\frac{l r_{*}}{2 r^{2}}\right) \cos \varphi, \\
e_{(\mathrm{in}) \varphi} \varphi^{\hat{x}}=-\left(1-\frac{l}{2 r_{*}}\right) r \sin \varphi, & e_{(\mathrm{ex}) \varphi} \varphi^{\hat{x}}=-\left(r+\frac{l r_{*}}{2 r}\right) \sin \varphi, \\
e_{(\mathrm{in}) r^{\hat{y}}}=\left(1-\frac{l}{2 r_{*}}\right) \sin \varphi, & e_{(\mathrm{ex}) r^{\hat{y}}}=\left(1-\frac{l r_{*}}{2 r^{2}}\right) \sin \varphi, \\
e_{(\mathrm{in}) r} r^{\hat{x}}=\left(1-\frac{l}{2 r_{*}}\right) r \cos \varphi, & e_{(\mathrm{ex}) r^{\hat{y}}}=\left(r+\frac{l r_{*}}{2 r}\right) \cos \varphi .
\end{array}
$$

We see that radial components $e_{r}{ }^{\hat{x}}$ and $e_{r}{ }^{\hat{y}}$ are continuous functions at the gluing surface $r=r_{*}$, while angular components have the jump:

$$
\begin{aligned}
& \left.e_{(\operatorname{ex}) \varphi}^{\hat{x}}\right|_{r=r_{*}}-\left.e_{(\mathrm{in}) \varphi}^{\hat{x}}\right|_{r=r_{*}}=-l \sin \varphi \\
& \left.e_{(\operatorname{ex}) \varphi} \hat{y}\right|_{r=r_{*}}-\left.e_{(\mathrm{in}) \varphi} \hat{\varphi}\right|_{r=r_{*}}=l \cos \varphi,
\end{aligned}
$$

The absence of disclinations in the media means that curvature of $\mathbb{S O}(3)$-connection vanishes. Therefore we put the connection components to zero without loss of generality. Afterwards, the torsion is defined entirely by the frame components

$$
T_{\alpha \beta}^{a}=\partial_{\alpha} e_{\beta}^{a}-\partial_{\beta} e_{\alpha}^{a} .
$$

For the tube dislocation they can be easily calculated. In the inside and outside regions, they are identically zero because elastic deformations are diffeomorphisms and cannot produce nontrivial geometry. At the gluing surface $r=r_{*}$ the frame jumps (3.2) and produce nontrivial torsion

$$
\begin{aligned}
& T_{r \varphi} \varphi^{\hat{x}}=-T_{\varphi r} \hat{x}=-l \sin \varphi \delta\left(r-r_{*}\right), \\
& T_{r \varphi} \hat{y}=-T_{\varphi r} \hat{y}=l \cos \varphi \delta\left(r-r_{*}\right),
\end{aligned}
$$

proportional to the $\delta$-function on the gluing surface. 
The torsion tensor is interpreted as the surface density of the Burgers vector in the geometric theory of defects. The total Burgers vector for a tube dislocation is zero. To get some feeling what happens with the Burgers vector for a tube dislocation, we compute it for a wedge shown in Fig.2. To compute the Burgers vector $\mathbf{b}=\left(b^{\hat{x}}, b^{\hat{y}}\right)$, we choose the contour of integration surrounding the

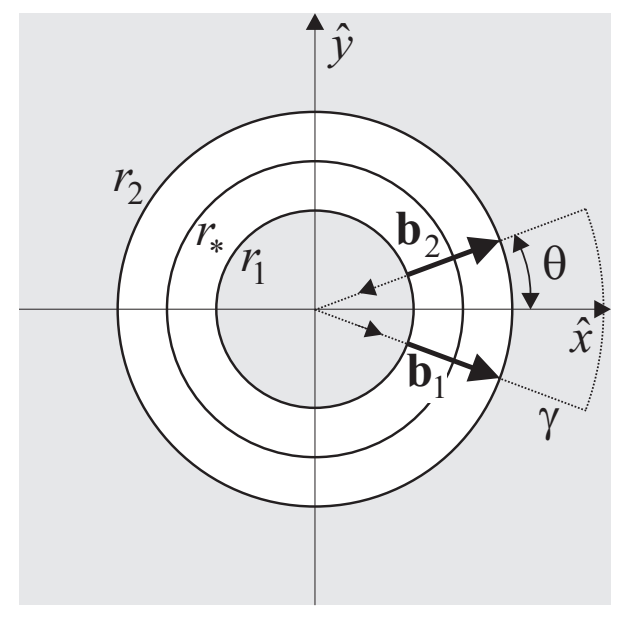

Figure 2: The Burgers vector for a wedge in a tube dislocation.

infinite wedge in the $x, y$ plane for $\varphi$ varying from $-\theta$ to $\theta$. Its components are given by the surface integral of the torsion tensor,

$$
b^{a}=\frac{1}{2} \int d x^{\alpha} \wedge d x^{\beta} T_{\alpha \beta}^{a} .
$$

For the tube dislocation, we obtain

$$
\begin{aligned}
& b^{\hat{x}}=\int_{0}^{\infty} d r \int_{-\theta}^{\theta} d \varphi T_{r \varphi}^{\hat{x}}=-l \int_{0}^{\infty} d r \delta\left(r-r_{*}\right) \int_{-\theta}^{\theta} d \varphi \sin \varphi=0, \\
& b^{\hat{y}}=\int_{0}^{\infty} d r \int_{-\theta}^{\theta} d \varphi T_{r \varphi} \hat{y}=l \int_{0}^{\infty} d r \delta\left(r-r_{*}\right) \int_{-\theta}^{\theta} d \varphi \cos \varphi=2 l \sin \theta .
\end{aligned}
$$

The first integral vanishes because $\sin \varphi$ is an odd function. This result is quite natural. By definition, the Burgers vector equals to the minus total jump of the displacement vector field along the contour of integration [8]. In the considered case, $\mathbf{b}=\mathbf{b}_{2}-\mathbf{b}_{1}$ where vectors $\mathbf{b}_{1}$ and $\mathbf{b}_{2}$ are shown in Fig.2. Thus the Burgers vector

$$
\mathbf{b}=(0,2 l \sin \theta)
$$

has only one nonvanishing component along $y$ axis because the wedge is chosen symmetrically. It coincides with the Burgers vector obtained within the elasticity theory.

\section{Conclusion}

The tube dislocation is a new exact solution of three dimensional Euclidean Einstein's equations with $\delta^{\prime}$ source. The corresponding metric components are discontinuous but all ambiguous terms in Einstein's equations cancel. This solution has straightforward physical interpretation in 
the elasticity theory as the tube dislocation. Defects result in nontrivial Riemann-Cartan geometry in the geometric theory of defects. In particular, dislocations are responsible for nontrivial torsion. Here we computed torsion components for a tube dislocation. These calculations are nontrivial because care must be taken in the definition of the triad field. Torsion components has physical meaning as the surface density of the Burgers vector in the geometric theory of defects. The Burgers vector for a tube dislocation is calculated and coincides with that in the elasticity theory.

This work is partly supported by the Russian Foundation of Basic Research (Grants No. 0201-01084 and 09-01-12161-ofi_m), and the Program for Supporting Leading Scientific Schools (Grant No. NSh-7675.2010.1).

\section{References}

[1] G. de Berredo-Peixoto and M. O. Katanaev. Tube dislocations in gravity. J. Math. Phys., 50:042501, 2009. gr-qc/0810.0243.

[2] G. de Berredo-Peixoto, M. O. Katanaev, E. Konstantinova, I. L. Shapiro. Schrödinder equation in the space with cylindrical geometric defect and possible application to multi-wall nanotubes. Nuovo Cim., 125B(8):915-931, 2010. arXiv:1010.2913[cond-mat.mes-hall]

[3] M. O. Katanaev and I. V. Volovich. Theory of defects in solids and three-dimensional gravity. Ann. Phys., 216(1):1-28, 1992.

[4] M. O. Katanaev and I. V. Volovich. Scattering on dislocations and cosmic strings in the geometric theory of defects. Ann. Phys., 271:203-232, 1999.

[5] M. O. Katanaev. Wedge dislocation in the geometric theory of defects. Theor. Math. Phys., 135(2):733-744, 2003.

[6] M. O. Katanaev. One-dimensional topologically nontrivial solutions in the Skyrme model. Theor. Math. Phys., 138(2):163-176, 2004.

[7] G. de Berredo-Peixoto and M. O. Katanaev. Inside the BTZ black hole. Phys. Rev. D, 75:024004, 2007. gr-qc/0611143.

[8] M. O. Katanaev. Geometric theory of defects. Physics - Uspekhi, 48(7):675-701, 2005.

[9] L. D. Landau and E. M. Lifshits. Theory of Elasticity. Pergamon, Oxford, 1970. 